\section{ATOMIC ENERGY AND ITS APPLICATIONS*}

\section{By SIR JAMES CHADWICK, F.R.S.}

$\mathrm{T}$ AE fission process can be briefly described as follows. A neutron is captured by a nucleus of uranium 235, forming a nucleus of uranium 236 in a highly excited state. This immediately divides into two fragments of roughly equal mass, and at the same time from one to three neutrons are emitted (and also some $\gamma$-radiation). The fragments fly apart with considerable speed and, in their passage through matter, their energy of motion is finally transformed into heat. These fragments are, in general, unstable and may pass through a series of transformations, emitting $\beta$-particles and $\gamma$-radiations, before they reach a stable configuration.

It was immediately recognized that this discovery was of the greatest significance and that it opened up the possibility of a practical utilization of the enormous store of atomic energy. Not only did the fission of uranium release a large amount of energy, but also the emission of more than one new neutron each time that a nucleus suffered fission suggested the possibility that the reaction could be propagated from atom to atom, developing a whole chain of reactions from one single event. Such a chain process developing in a mass of uranium might take place at an ever-increasing rate and might involve so many atoms that there would be an appreciable, and possibly an overwhelming, liberation of energy. In this way it seemed that a new type of combustion of matter might be possible which would give an energy yield a million times greater than that obtainable by the combustion of the same weight of coal or oil.

A chain reaction will not necessarily develop in any piece of fissile material. Certain conditions must be satisfied. In the first place, the amount of fissile material, or the reacting system as a whole, must be of such a size that there is not too great a chance that the neutrons produced in the fission process can escape and so be unable to carry on the chain. Secondly, the system must not contain more than a limited amount of material which absorbs neutrons, for in this way again the neutrons will be prevented from continuing the chain reaction.

If these conditions are satisfied, the chain reaction will develop and 'run away', unless some special device is introduced to control it. Thus we have two general types of chain reaction-the uncontrolled reaction and the controlled reaction.

\section{Uncontrolled Reaction: the Atomic Bomb}

If we wish to use atomic energy as an explosive the reaction must be uncontrolled, for the more rapidly it can proceed, the greater will be the amount of energy set free and the explosive effect.

The question of size is critical for an atomic explosion, for a chain reaction cannot develop if the mass of fissile material is too small. This is because the reaction depends on the conservation of the neutrons produced in the fissions. If the piece of material is too small or of an unsuitable shape, too many neutrons will escape before they hit a nucleus and propagate the reaction. The proportion of neutrons which escape can be reduced by increasing the size of the piece, since the production of neutrons

* From the Melchett Lecture of the Institute of Fuel delivered on October 8. The full lecture is published in the Journal of the Institute of Fuel, Dec. 15, 1946 . is a volume effect and will therefore increase more rapidly with size than the loss of neutrons by escape, which is a surface effect. Thus a certain minimum amount of material is required, called the critical size or amount, before the reaction can develop so fully that an explosion occurs. If the piece of fissile material is less than the critical size it will be quite stable; but as soon as it exceeds this a chain reaction will develop and multiply itself with enormous rapidity. Thus all that is necessary to detonate a bomb of uranium 235 is to bring together two pieces each less than the critical size but which when in contact exceed it.

Suppose we have succeeded in doing this by some means, and that at the same moment we shoot a neutron into the material. This will cause fission of one uranium nucleus and produce, let us say, two fission neutrons which in turn produce four, and so on in successive generations. Since the fission neutrons have speeds of the order of $10^{\circ} \mathrm{cm}$. per sec., and since they do not have to travel many centimetres before they produce new fissions, the time between successive generations, in which the number of neutrons is doubled, is of the order of $10^{-8} \mathrm{sec}$. In a millionth of a second, therefore, the number of neutrons will have increased enormously and a considerable fraction of the mass of U235 will have undergone fission. If only $1 \mathrm{kgm}$. is used up in this way, the amount of energy released will be as much as from 17,000 tons of T.N.T. This amount of energy is so great that the mass will attain a temperature of many million degrees and a pressure of many million atmospheres. The high temperature causes the emission of an enormous amount of heat and light, and the sudden expansion of the hot gases produces the shock wave of the explosion.

As the material expands, the neutrons can escape more easily and the reaction stops. By this time the hot glowing gases may have expanded to a diameter of about a third of a mile.

In addition to the blast wave and the intense heat and light from the hot gases, the atomic bomb emits an enormous radiation of gamma rays and neutrons at the moment of explosion. Further, the products formed by the fission process are intensely radioactive, and the total amount formed in the explosion may correspond in activity to many tons of radium.

\section{Control of the Chain Reaction}

In order to use atomic energy for industrial purposes, it is obviously necessary to control the chain reaction so that the energy can be released continuously and at a predetermined rate. It seems at first sight that the control of a reaction which can develop so rapidly and which can liberate such enormous energies must be exceedingly difficult. This is, fortunately, not the case, and in practice the control is remarkably simple, effective and accurate.

Control depends on the regulation of the neutron flux so that at a predetermined level the flux remains constant, only exactly as many neutrons causing fission in one generation as in the previous generation. The extra neutrons which are produced in the fissions are removed and made unavailable for the reaction. The reaction will then proceed at a constant rate. This balance can be achieved either: (1) by allowing the excess neutrons to escape from the system ; or (2) by capturing them in some absorbing medium. 
It will be convenient at this point to define a quantity called the multiplication or reproduction factor. Let us suppose that in a system containing fissile material there is a certain number, $P$, of free neutrons present at a given time. Some of these neutrons will initiate fissions and thus directly produce new neutrons, $Q$ in number. The ratio $Q / P$ is called the multiplication factor and denoted by $k$.

If $k$ is less than 1 , no chain reaction can develop. Any stray neutrons may produce a fission, but the chain will die away quickly and the system will be perfectly safe. This corresponds to a system which contains less than the critical amount of fissile material.

If $k$ is greater than 1 , as in the example quoted above for the atomic bomb, the number of neutrons will increase rapidly from generation to generation and an explosion will occur.

When $k$ is exactly equal to 1 , the reaction will proceed at a constant rate and the flux of neutrons will remain constant. This is the condition which has to be achieved for successful application of the chain reaction to general purposes.

The principle of the control of a chain reactor can be illustrated by a simple example. Let us suppose that we have two masses, $A$ and $B$, of fissile material, each less than the critical amount but together considerably greater than it, and let us consider what happens as $A$ and $B$ are brought closer together.

When $A$ and $B$ are far apart, the leakage of neutrons will be great and the multiplication factor $k$ will be less than 1. Suppose that at some stage in the approach $k$ becomes equal to 0.99 and that we have in the material a source of neutrons which emits 1 neutron per second. This one neutron will produce $k$ neutrons in the first generation, these $k$ will produce $k^{2}$, and so on, so that the total number of neutrons being produced per second will be :

$$
1+k+k^{2}+k^{8} \ldots=\frac{1}{1-k}
$$

if $k$ is less than $\mathbf{I}$.

Thus when $k=0.99$ there will be 100 neutrons produced per second in the material, and the primary source has been multiplied 100 times. As the two parts are brought closer and closer together the leakage of neutrons diminishes, $k$ becomes correspondingly nearer and nearer to 1 , and the neutron intensity can be brought to any desired value.

It is evident, however, that an exceedingly fine adjustment of $k$ will be required to reach a high intensity, so fine as to be impossible as a practical operation.

Let us now suppose that the two parts are close enough to make $k$ slightly greater than 1. A divergent chain reaction will now develop and the neutron intensity will rise rapidly, doubling itself in each successive interval of time $T$ given by the formula

$$
T=0.7 \frac{\tau}{k-1}
$$

where $\tau$ is the average life-time of one generation of neutrons.

Thus by making $k$ slightly greater than 1 we could raise the level of neutron intensity very rapidly and, having reached the level it is desired to have in our reactor, we could now reduce $k$ to 1 so that the desired level would be maintained.

While this adjustment is simple in principle, the possibility of carrying it out depends very much on the value of $\tau$. Now the fission neutrons are emitted with velocities of the order of $10^{9} \mathrm{~cm}$. per sec., so that if they were all emitted instantaneously upon fission the value of $\tau$ would be of the order of $10^{-8}$ sec. But in order to allow time for the adjustment of the mechanisms which control the positions of $A$ and $B$, we need a doubling time $T$ of the order of a second; and this would entail an adjustment of the value of $k$ to less than one hundred-millionth part of its value. Even if this could be achieved, there would always be the danger of a slight temperature change or mechanical disturbance which might cause the neutron intensity to rise at an uncontrollable rate and result in a violent explosion.

If the picture I have given were quite accurate, the control of the chain reaction would evidently be a formidable operation. Fortunately, however, not quite all the neutrons are emitted instantaneously upon fission. Some of the fission products transform after a time with the emission of a $\beta$-particle into nuclei which are now unstable and emit neutrons.

We have, therefore, in addition to the prompt neutrons released in the fission process itself some neutrons which are delayed after the fission by the time required for the transformation of the fission fragments: The effect of these delayed neutrons is to increase the average time for neutron generation and, therefore, to reduce the rate at which the chain reaction develops.

The importance of this effect can be shown by a simple calculation.

For the purpose of this calculation we may assume that the delayed neutrons form 1 per cent of the total neutron emission from fission and that the average time of delay is 10 sec. We now arrange the assembly of fissile material so that it is only just above the critical condition, with a multiplication factor $k$ greater than 1 but less than $1 \cdot 01$. This means that the prompt neutrons alone will be insufficient to maintain the chain reaction and each generation of neutrons will have to wait for the delayed neutron emission before a divergent increase in intensity can occur. The neutron intensity will double itself for each interval of time $T$ given by

$$
T=0.7 \cdot \frac{1-k_{p}}{k-1} \tau_{D}
$$

where $k_{p}$ is the multiplication factor due to the prompt neutrons alone, and $\tau_{D}$ is the average time between the fission and the emission of the delayed neutrons, that is, $10 \mathrm{sec}$.

If we put $k=1 \cdot 005$, then $k_{p}$ will be about 0.995 , and the doubling time is about 7 sec.

Thus, provided the assembly is only a small degree above the critical size, the effect of the delayed neutron emission is to make the reaction grow quite slowly. It will therefore be easy to control the reaction and to hold the level of the reaction and the neutron intensity at any desired level. The adjustment of the neutron leakage may be a simple type of mechanical control which moves a small part of the reactor relative to the main system or which moves reflectors which can reflect back into the system some of the neutrons which would otherwise escape. In this way, the reaction can be controlled smoothly and the level of the reaction can be held very steady ; in fact, in some of the so-called 'slow reactors', the level can be held to better than one part in 10,000 over long periods of time. 


\section{Fast and Slow Neutron Reactors}

The systems I have so far considered are known as fast neutron reactors, because the chain reaction is carried on by the fission neutrons without moderation of their speed except that which inevitably must occur in collisions in the system, whether in the fissile material itself or in parts of the apparatus for assembly, etc., and these neutrons have velocities of the order of $10^{9} \mathrm{~cm}$. per sec.

Slow neutrons, however, are much more effective in producing fission both in U235 and in plutonium than are fast neutrons. Thus, if the fission neutrons could be slowed down before reproducing themselves, the chain reaction could be maintained in a smaller amount of fissile material, or even in much less pure material than is required for the fast reactor.

One advantage of the slow neutron reactor is the simplicity of control. Some substances, notably boron and cadmium, absorb slow neutrons very strongly. This fact provides a very ready method of controlling the reaction, by moving rods of these materials in or out of the reactor and so adjusting the multiplication factor by changing the loss of eutrons by absorption rather than by changing the )ss by leakage as in the fast reactor. It is obvious lat a simple mechanical device will suffice to carry ut this control.

A very important advantage offered by the use of ow neutrons is that it is no longer necessary to use ure or nearly pure fissile material in order that the chain reaction should maintain itself. In fact, as is well known, it is possible to use natural uranium, thus avoiding the very expensive and tedious process of separating the isotopes.

In this case, owing to the fact that the 238 isotope captures neutrons without undergoing fission and that this isotope is 139 times as abundant as the fissile 235, the choice of material for slowing down the neutrons is rather limited, for very little additional loss of neutrons can be tolerated. For this reason it has not been found possible to use water as moderator, for hydrogen captures slow neutrons too readily. Heavy water makes a very suitable moderator, for the capture by deuterium is small, and it has been successfully used in the United States and later in Canada. It is, however, too expensive a substance to use for large reactors.

The best material readily available at the present time is carbon, in the form of graphite. This has the advantage of being relatively cheap and abundant, and it can be obtained in a very pure state.

Owing to the inevitable, though small, loss of neutrons by absorption in the graphite, the multiplication factor of a uranium-graphite system is not much greater than 1. The critical size of the reactor is, therefore, rather large, and some tons of uranium and of graphite are required. The construction of a reactor of small power is a straightforward matter, but difficulties arise when large power dissipation.is desired, of the order, say, of $100,000 \mathrm{~kW}$., owing to the small latitude in the multiplication factor. The nature of these difficulties will be best illustrated by a brief description of the large reactors, the so-called graphite piles, erected at Hanford, although the primary purpose of these piles was the production of plutonium rather than the generation of power.

\section{Natural Uranium Reactor-the Graphite Pile}

For reasons depending on the nuclear properties of uranium, the arrangement which gives the highest multiplication factor is one in which lumps of metallic uranium are disposed at regular intervals throughout a large block of graphite in the form of a lattice. The ideal shape would be spherical, but for practical considerations a rectangular block is used. The optimum size of the uranium lumps and of the lattice spacing can be calculated from the nuclear constants of the materials. This was the arrangement used in the first graphite pile of very low power, but it had to be modified for the plutonium production pile. There were two reasons : first, the uranium has to be cooled in order to remove the heat generated by fission, and it is difficult to concentrate the coolant at small lumps, and second, it was required to remove the uranium without disturbing the assembly of the pile.

The arrangement used in the Hanford piles is, therefore, a rod lattice of cylindrical symmetry, in which short rods or slugs of uranium are loaded in cylindrical channels in the graphite moderator. This entails some reduction in the multiplication factor.

The cooling agent, water in this case, is circulated through pipes around the rods. Since graphite pipes are not practical, on engineering grounds, some other material has to be used, and the choice is limited by the condition that the material must have a small absorption for neutrons. The material chosen was aluminium.

Further, the uranium must be protected from the cooling water, for it is easily oxidized, and even if the slugs were not disintegrated by chemical action, sufficient fission products would enter the cooling water to make operation dangerous. The protective jacket must have a high conductivity in order to transmit heat quickly from the uranium to the water, it must not absorb too many neutrons, and it must not corrode. Aluminium fulfils these conditions fairly well.

The introduction of this auxiliary material in the form of cooling pipes, sheathing, instruments, etc., increases the loss of neutrons by absorption, and it is, therefore, necessary to keep down the loss by leakage to a few per cent. This is not difficult, for the leakage can obviously be made as small as desired by making the reacting system sufficiently large.

The problem of dissipating some $100,000 \mathrm{~kW}$. or so is not difficult provided an ample supply of pure water is available, but it is complicated by factors peculiar to an atomic energy reactor. In its passage through the pile, the water becomes radioactive, so that if the water is to be returned to a river a retention basin must be provided to ensure a delay, during which the induced radioactivity dies down to a safe level. Further, the circulating system must be very reliable, and fast-operating controls must be provided in order to shut down the chain reaction quickly in case of failure of the water supply.

The control is essentially no more difficult than for a small slow reactor, but, of course, the large reactor is potentially much more dangerous. The operating controls, connected with instruments in the pile, which measure the neutron density and thus the power level, can automatically keep the pile operating at a predetermined level. In addition, emergency controls are provided to operate as soon as the power level shows signs of undue increase.

The radiation from a pile operating even at low power is strong enough to be dangerous to human life, and that from a high-power pile reaches a fantastic intensity. The whole of the reacting unit must, therefore, be enclosed in steel and thick walls of 
concrete, and it must be possible to load and unload the pile through these shields. Any operation on the pile must be carried out by remote control.

The design and operation of the Hanford piles was thus beset by difficulties, many of them new and peculiar to an atomic energy plant, but they have operated for nearly two years without trouble at a power level of perhaps a few hundred thousand kilowatts.

The total weight of a pile, including the massive shielding, amounts to some thousands of tons, but it is, nevertheless, a very compact unit for the amount of power which is generated in it.

\section{Production of Useful Power}

The energy generated in the Hanford piles is removed by the cooling water which issues at a temperature considerably below $100^{\circ}$ C.-too low a temperature for efficient conversion of the heat to useful power. If such a pile is to have general industrial applications, such as the generation of steam and the generation of electric power, it must be run at a much higher temperature. To do this will necessitate considerable changes in design and in operating technique.

It would probably be possible to use liquid bismuth as coolant instead of water, and it might be an advantage to use some other material than graphite as moderator. Progress on these lines demands the solution of some awkward engineering and metallurgical problems; but there is no reason to suppose that the difficulties cannot be overcome.

The most striking characteristics of atomic energy reactors are their compactness for large power capacity and their ability to run for long periods with little or no refuelling.

The power available from an atomic energy reactor is limited essentially only by the rate at which the heat can be removed from it, so that a million-kW. plant need be no bigger than a normal electric power sub-station.

The energy released in the combustion by fission of $1 \mathrm{lb}$. of U235 or plutonium is rather more than 11 million $\mathrm{kWh}$., equal to the amount of energy which can be obtained from about 1,500 tons of coal or 250,000 gallons of fuel oil. Thus an atomic energy unit may be able to run for long periods on one charge of fuel, and the cost of transport of fuel will, in any event, be small.

Moreover, the plant may, under appropriate conditions, be able to regenerate some, if not all, of its fuel. This arises from the possibility of designing the plant so that some of the neutrons released in fission are used to produce plutonium in uranium, disposed in a suitable way inside or outside the reactor, which will replace the U235 or plutonium which is burned.

This process of replacement takes place in the Hanford piles, where U235 is indirectly converted into plutonium. It may be possible under some conditions to arrange that as uranium 235 is burned the reaction could continue by burning the plutonium, and in this way most of the uranium 238 as well as the 235 might be utilized.

This relative freedom from supply problems, once the plant is set up, opens up the possibility of stimulating industrial development in isolated parts of the world, where coal- or water-power are not readily available. For the same reason, it might prove advantageous to establish atomic energy units in close proximity to mines, more especially in those cases where the ores have to be transported long distances in their crude states.

They may also find application in large oceangoing vessels, in cases where it is desired to avoid frequent refuelling. This application is somewhat more questionable on account of some aspects of atomic reactors, to which I shall refer later.

We must not forget, however, that these bright prospects for the peaceful applications of atomic energy depend on the solution of difficult engineering problems, and that no reactor has yet been developed, or, I believe, even designed, which is suitable for the purposes I have mentioned. Nor must we forget the limitations and disadvantages to which the atomic energy unit is subject, and which are inherent to it.

One of the most serious disadvantages from the point of view of general industrial use arises from the fact that an atomic energy reactor is a source of very intense and very dangerous radiations. A reactor operating at a power level of 1 watt produces as many $\gamma$-rays as a few grams of radium (without the radiations from the fission products) and some neutron radiation as well. Although a considerable fraction of these radiations will be absorbed in the reactor itself, shielding is required in order to protect personnel in the vicinity. Even in the case of a very compact unit, the weight of shielding will be con. siderablé.

For example, let us take the case of a reactor dissipating (not generating as useful energy) 100,000 $\mathrm{kW}$. This cannot be much less in size than a $\mathrm{I}$. metre cube, having regard to the difficulty of the problems of heat transfer. It is easy to show that the weight of the shielding required to reduce the radiations from this unit to a safe level would be a few hundred tons. Even a low-power reactor will need shielding at least 50 to 100 tons in weight, and this factor alone prohibits the application of this type of power to motor-cars or any small mobile units.

Another limitation in the application of atomic energy is that a relatively large amount of fissile material is required even for a low-power unit. This arises from the fact that a 'critical size' is necessary before the chain reaction can develop. Thus, for some time to come, the minimum cost of materials for a power unit may be many thousands of pounds, and it becomes uneconomic to use atomic energy for small plants.

Another consideration is that practically every material which is incorporated in the construction of an atomic reactor becomes radioactive owing to its exposure to neutrons. For this reason, special provisions would have to be devised for maintenance.

The intense $\gamma$ - and neutron-radiations also have an effect on the structural properties of the materials entering into the construction of the reactor. Owing to their ionizing properties, these radiations destroy the molecular bonds of many molecules. The choice of substances which can be used is, therefore, restricted to those which tend to return to their original state after intense ionization, such substances as elements and simple ionic compounds. How far even these will endure the very great irradiations resulting from large power outputs is not known, for experience is still very meagre. This effect may possibly set a limit to the power output of an atomic reactor and even to its life.

A further difficulty may be caused by the fission products, more especially in the case of the natural uranium reactor. Some of these fission products may absorb neutrons so strongly that after some time the 
chain reaction will be inhibited. In such a case, it would be necessary to remove the uranium, extract the fission products, and either to recharge the reactor with new metal or to recover the uranium and to replace it, adding sufficient plutonium or U235 to make it reactive.

Of necessity, all reactors manufacture fission products. At some stage or other, it will ultimately be desirable to remove them. Their disposal may prove to be an awkward problem.

\section{Cost of Power Derived from Atomic Energy}

So far as the immediate future is concerned, the application of atomic energy to the production of power is not likely to result in a net decrease of overall costs to the community.

Owing to the difficult and tedious nature of the processes for preparing U235 or plutonium in high concentration, reactors depending on such materials for fuel will be far more costly to run than existing hydro-electric or steam-power plants, and the most favourable comparison at the present time will be given by the natural uranium reactor.

Recently an American committee has made a careful study of the costs of building and operating a natural uranium reactor to deliver $75,000 \mathrm{~kW}$., and has concluded that the over-all cost of powes would be only about 25 per cent greater than for a coalburning power plant. This estimate is based on the assumption of a high operating temperature of the uranium reactor, that is, on the performance of a plant which has not even been designed, let alone built. It does not, therefore, represent the present position, but the possible position in a few years time, when research and development have provided the solution of a number of difficult technical problems. The committee's estimate is nevertheless valuable in indicating the possibilities which can already be foreseen. It is not unreasonable to expect that future development of atomic energy will result in a material reduction of costs, and perhaps make the uranium reactor a direct competitor with other power plants.

At the present moment it seems that the use of atomic power plants would be justified, on a purely economic basis, only in the special circumstances I have previously mentioned, where the power needed to develop resources is either very costly or unavailable, or because of difficulties of transport.

What is needed now is intensive research and development in the technical problems which are involved in the applications of atomic energy. This will not only clarify our present somewhat vague ideas, but it may well be that quite new lines may be opened up.

\section{The Future}

In assessing the possibilities of future development it is well to remember not only that the art is in its infancy, but also that the preoccupation of the war years with the military aspects of atomic energy has excluded almost completely the consideration of industrial applications. These military aspects still have, and, until some far-reaching international agreement on the control of such weapons is obtained, are bound to have, a retarding influence on peaceful developments, for the reason that the paramount need for security prevents that wide dissemination of knowledge which promotes free exchange of thought, stimulates discovery, and gives an impetus to invention and development.
At this early stage in our knowledge of these matters one cannot hope to see clearly into the future, but only to discern the probable lines of progress. Upon further investigation some lines we now have in mind may prove fruitless, but if we can be guided by the history of scientific and technical development, it is more than likely that others will give a rich reward. A few short years ago what has even now been accomplished would have been considered an idle dream.

Already a military weapon of unparalleled power has been created; enormous amounts of radioactive material have been manufactured; substances which have, owing to their inherent instability, become very rare or even completely disappeared from the cupboard of Nature, have been built up by individual nuclear transmutations and collected in large quantities; and the first steps have been taken towards the development of these new energy sources for industrial application.

This new enterprise constitutes a far deeper interference with the natural course of events than anything which has been attempted hitherto, and it is a most remarkable example of the powers of physical science and engineering and of our increasing control over the processes of Nature. It is not easy at this early stage to survey in detail the consequences which may flow from this advance in the years to come, but it can scarcely fail in the long run to bring about a new outlook and a new situation as regards human resources.

\section{DR. JAMES HUTTON AND THE MANUFACTURE OF SAL AMMONIAC}

\section{By ARCHIBALD CLOW and NAN L. CLOW}

THE hundred and fiftieth anniversary of the death of Dr. James Hutton (1726-97), the celebrated geologist, which occurred on March 26, brings to mind a little-known phase in the utilization of the products of coal distillation, the profits from which are said to have secured to Hutton the leisure necessary for his geological speculations.

In his "History of Inventions and Discoveries", J. Beckmann says : "If I am not mistaken, the first manufactories of sal ammoniac were established in Scotland: the oldest of these, perhaps, was that erected by Dovin and Hutton at Edinburgh in 1756 "'.

This refers to a co-partnership formed by Dr. James Hutton and someone variously described as James Dovin, Dairy, or Davie, in Edinburgh according to a plan conceived when they were Edinburgh students. In Kay's "Portraits", the date of formation is given as before 1749, which may be right, since Hutton went to a Norfolk farm to study agricultural methods in 1752 .

The raw material used in this early chemical manufacture was soot, and for a number of years Davie took all the soot collected by Edinburgh 'tronmen'- - a chimney sweeps' society formed in $1738^{3}$. The process was carried out as follows: "Globular glass vessels, about a foot in diameter, are filled to within a few inches of their mouth with it [soot], and are then arranged in an oblong furnace, where 